\title{
Fatty acid desaturation-defective mutants of an arachidonic-acid- producing fungus, Mortierella alpina 1S-4
}

\author{
Saeree Jareonkitmongkol, Sakayu Shimizu* and Hideaki Yamada \\ Department of Agricultural Chemistry, Kyoto University, Sakyo-ku, Kyoto 606, Japan
}

(Received 26 November 1991; revised 23 January 1992; accepted 11 February 1992)

\begin{abstract}
Three mutants, which were defective in the desaturation of fatty acids, were isolated from an arachidonic-acidproducing fungus, Mortierella alpina 1S-4, after treating wild-type spores with $N$-methyl- $N^{\prime}$-nitro- $N$ nitrosoguanidine. They were designated Mut44, Mut48 and Mut49. Mut44 was a mutant with low $\Delta 5$-desaturase activity. It accumulated a high level of dihomo- $\gamma$-linolenic acid (DGLA) $(28.6 \%$, $w / w)$ but a low level of arachidonic acid (Ara) (10.6\%), compared with the wild type, which had levels of 6.3 and $47.0 \%$, respectively. Mut48 was unable to desaturate oleic acid (18:1) to linoleic acid (18:2), i.e. $\Delta 12$-desaturation, and therefore a large amount of 18:1 (49.5\%) accumulated and no fatty acid of the $\omega-6$ family was detected. In addition, several fatty acids of the $\omega-9$ family, such as 5,8,11-cis-eicosatrienoic acid, were found. In Mut49, 18:2 (46\%) accumulated markedly, but only small amounts of DGLA and Ara were detected. Thus, Mut49 was considered to be defective in $\Delta 6$-desaturation. These mutants showed a somewhat longer lag phase than the wild type on cultivation at both 28 and $12{ }^{\circ} \mathrm{C}$.
\end{abstract}

\section{Introduction}

During the course of a study on the production of polyunsaturated fatty acids (PUFAs), such as dihomo- $\gamma$ linolenic acid (DGLA), arachidonic acid (Ara), 5,8, 11,14,17-cis-eicosapentaenoic acid (EPA) and so on, by micro-organisms, we have found that many members of the Mortierella subgenus, especially the soil isolate Mortierella alpina 1S-4, are potent producers of these PUFAs (Yamada et al., 1987; Shimizu et al., 1988a,b, 1989; Shinmen et al., 1989). However, how these PUFAs are produced has remained unclear. In order to obtain further details on their biosynthesis by this fungus, with a view to the possibility of increasing their production, we concentrated on the isolation of PUFA biosynthesis mutants.

In this paper, we report the isolation and character-

* Author for correspondence. Tel. 075753 6122; fax 81757536128.

Abbreviations: Ara, arachidonic acid; DGLA, dihomo- $\gamma$-linolenic acid; EPA, 5,8,11,14,17-cis-eicosapentaenoic acid; MNNG, $N$-methyl$N^{\prime}$-nitro- $N$-nitrosoguanidine; PC; phosphatidylcholine; PE, phosphatidylethanolamine; PS, phosphatidylserine; TG, triglycerides; $18: 2 \omega 9,6,9$-cis-octadecadienoic acid; $18: 3 \alpha, \alpha$-linolenic acid; $18: 3 \gamma$, $\gamma$-linolenic acid; $18: 4,6,9,12,15$-cis-octadecatetraenoic acid; $20: 2 \omega 9$, 8,11-cis-eicosadienoic acid; $20: 3 \omega 3,11,14,17$-cis-eicosatrienoic acid; $20: 3 \omega 9,5,8,11$-cis-eicosatrienoic acid; $20: 4 \omega 3,8,11,14,17$-cis-eicosatetraenoic acid. ization of three mutants of $M$. alpina $1 \mathrm{~S}-4$, which are considered to be completely defective (or to have low activities) in $\Delta 5-, \Delta 6-$ and $\Delta 12$-desaturation, respectively.

\section{Methods}

Chemicals. $N$-Methyl- $N^{\prime}$-nitro- $N$-nitrosoguanidine (MNNG) and triolein were obtained from Nacalai Tesque. Phospholipids were obtained from the Serdary Research Latoratory, London, Canada. All authentic fatty acids were obtained from Sigma except for Mead acid, which was purchased from Biomol Research Laboratories, Plymouth Meeting, PA, USA. All other reagents were of analytical grade.

Mutagenesis with MNNG. M. alpina 1S-4 (Yamada et al., 1987. Shimizu et al., $1988 b)$ was inoculated onto Czapek agar medium $(0.2 \%$ $\mathrm{NaNO}_{3}, 0.1 \% \mathrm{~K}_{2} \mathrm{HPO}_{4}, 0.05 \% \mathrm{MgSO}_{4} .7 \mathrm{H}_{2} \mathrm{O}, 0.05 \% \mathrm{KCl}, 0.001 \%$ $\mathrm{FeSO}_{4} .7 \mathrm{H}_{2} \mathrm{O}, 3 \%, \mathrm{w} / \mathrm{v}$, sucrose and $2 \%, \mathrm{w} / \mathrm{v}$, agar, pH 6.0) and allowed to sporulate at $28{ }^{\circ} \mathrm{C}$ for 2 weeks. Spores were collected by pouring $50 \mathrm{ml}$ sterile water containing a drop of Tween $\mathbf{4 0}$ into the medium, followed by filtration through 4-layers of gauze and centrifugation of the filtrate at 5000 r.p.m. for $10 \mathrm{~min}$. The spores thus obtained were suspended in $50 \mathrm{~mm}$-Tris/maleate buffer ( $\mathrm{pH} 7.5)$ at a concentration of approximately $1 \times 10^{6} \mathrm{ml}^{-1}$ and then exposed to MNNG $\left(100 \mu \mathrm{g} \mathrm{ml}^{-1}\right)$ at $28^{\circ} \mathrm{C}$ with reciprocal shaking for $20 \mathrm{~min}$. This resulted in survival rates of $0.1-5 \%$. Three millilitres of $10 \%(\mathrm{w} / \mathrm{v})$ sodium thiosulphate was added, followed by centrifugation at 5000 r.p.m. for $10 \mathrm{~min}$ and two washes with sterile distilled water.

Isolation of mutants. The MNNG-treated spores were inoculated into medium GY $(2 \%, w / v$, glucose and $1 \%, w / v$, yeast extract, pH 6.0) and then incubated at $12^{\circ} \mathrm{C}$ (or 6 or $15^{\circ} \mathrm{C}$ ) for $2-3 \mathrm{~d}$. Each culture was 


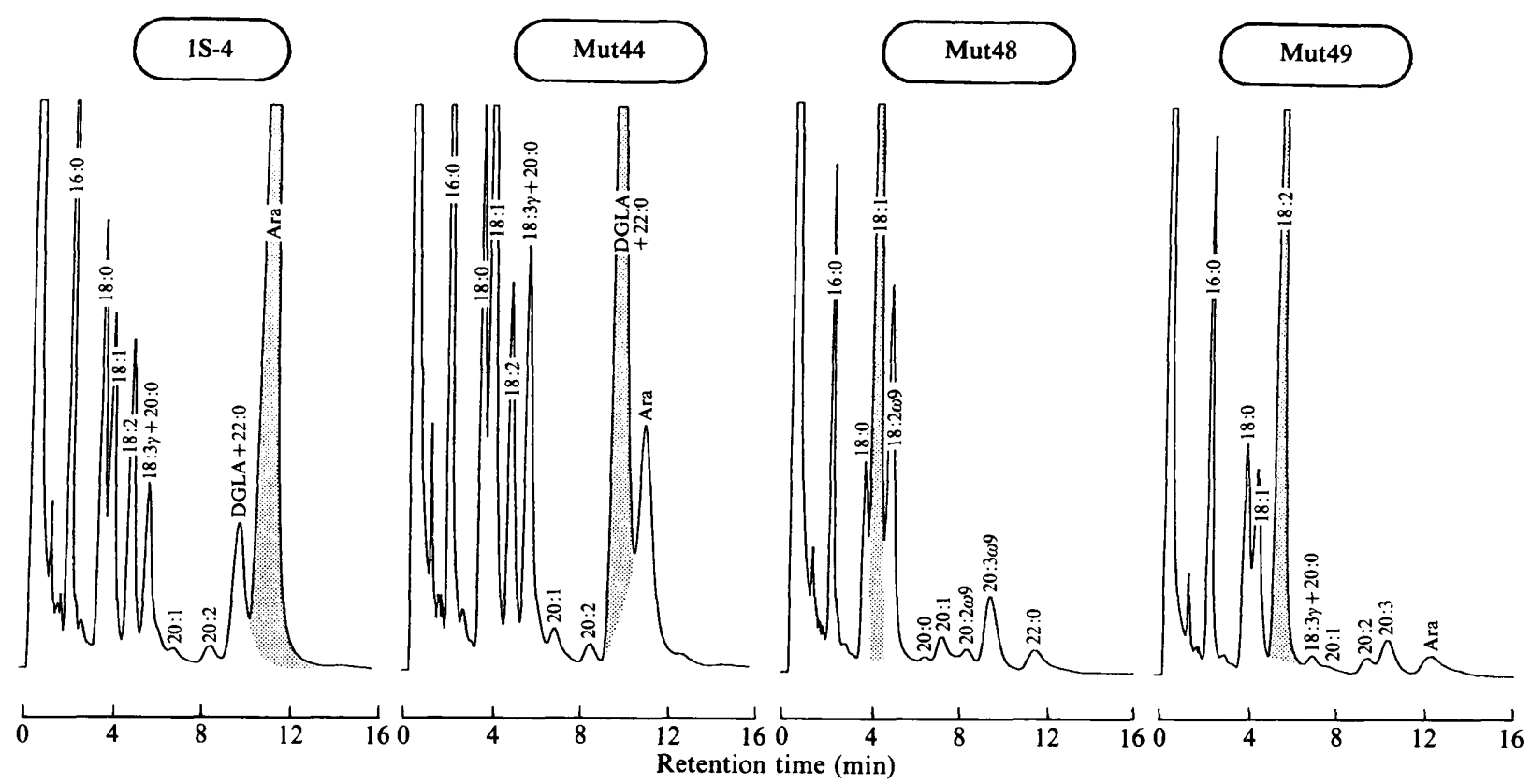

Fig. 1. Gas chromatograms of the fatty acid methyl esters obtained from $M$. alpina 1S-4 (wild type) and mutants Mut44, Mut48 and Mut49, defective in $\Delta 5-, \Delta 12-$ and $\Delta 6$-desaturation, respectively. The shaded peaks indicate the most abundant components.

filtered through a glass filter (pore diam., 20-30 $\mu \mathrm{m}$ ) and the filtrate was then centrifuged to collect the non-germinated spores (about $1-10 \%$ of the total treated spores). The spores thus obtained were then plated on a Petri dish (90 mm diam.) containing an agar medium (1\% glucose, $0.5 \%$ yeast extract, $0.005 \%$ Triton $\mathrm{X}-100$ and $2 \%$ agar), followed by incubation at $28{ }^{\circ} \mathrm{C}$ for $2 \mathrm{~d}$, when colonies appeared. About 100 colonies for each mutagenesis were randomly picked up and each of them was grown in liquid culture as described below. After incubation at $28^{\circ} \mathrm{C}$ for $5 \mathrm{~d}$, the cells were harvested, dried at $100^{\circ} \mathrm{C}$, and their fatty acid compositions analysed by GLC on a glass column $(3 \mathrm{~mm} \times 2 \mathrm{~m})$ packed with $5 \%$ Advanced DS on 80/100 mesh Chromasorb W (Nishio Kogyo). The column temperature was $190^{\circ} \mathrm{C}$ and other conditions were as given in detail previously (Shimizu et al., 1991a).

Liquid culture. M. alpina 1S-4 (or a mutant) was inoculated as a spore suspension into a $10 \mathrm{ml}$ Erlenmeyer flask containing $2 \mathrm{ml}$ medium GY, and then the culture was incubated at $28^{\circ} \mathrm{C}$ with reciprocal shaking $\left(120\right.$ strokes $\min ^{-1}$ ) for 1 week, unless stated otherwise. The mycelial cells were harvested by suction filtration, washed with distilled water, and then dried at $100^{\circ} \mathrm{C}$ overnight for subsequent fatty acid analysis by GLC as described above.

Lipid analysis. Extraction and fractionation into triglycerides and phospholipids were performed as described previously (Shimizu et al., $1991 a$ ), except that the bands of lipids were detected under ultraviolet light $(366 \mathrm{~nm})$ after spraying with $0.01 \%$ premulin dissolved in acetone-water $(4: 1, v / v)$, and that methyl heptadecanoate was used as an internal standard.

Other methods. Fungal growth was measured by determining the mycelial weight after drying at $100^{\circ} \mathrm{C}$ overnight. Fatty acids were identified by comparing their retention times on GLC with those of authentic standards. When authentic standard fatty acids were not commercially available, the fatty acids were isolated by reverse phase HPLC (Shimizu et al., 1991 a) and their structures were determined on the basis of both proton NMR and mass spectra of the corresponding picolinyl esters prepared as described by Christie et al. (1986). The conditions for mass spectrometry and proton NMR were as described previously (Shimizu et al., 1991a).

\section{Results}

\section{Selection and characterization of mutants}

Based on the assumption that mutants defective in fatty acid desaturases would be low-temperature sensitive, as in the case of the fatty acid desaturation-defective mutants of the cyanobacterium Synechocystis PCC6803 (Wada \& Murata, 1989), we focussed on the isolation of low temperature sensitive mutants. In order to increase the proportion of low temperature sensitive mutants, MNNG-treated spores were first incubated in medium GY at various temperatures lower than the physiological one for the wild type $\left(28^{\circ} \mathrm{C}\right)$, as described in Methods. On testing about 1300 colonies, we found three such mutants, designated Mut44, Mut48 and Mut49. These mutants were not distinguishable from the wild type in morphology, but their fatty acid profiles were apparently different from that of the wild type (Fig. 1). Mut44 accumulated a significantly high percentage content of DGLA $(28.6 \%$, w/w) but a low Ara content $(10.6 \%)$, compared with those in the case of the wild type, which were 6.3 and $47.0 \%$, respectively. This phenomenon was similar to that previously reported for the inhibition of desaturation of DGLA to Ara by sesamin, that is, 
Table 1. Fatty acid profiles of Mut48 and Mut49 grown in medium supplemented with fatty acids

Cells were cultured in duplicate in a medium containing $1 \%$ glucose and $0.5 \%$ yeast extract ( $\mathrm{pH} 6.0$ ) at $28{ }^{\circ} \mathrm{C}$ for $1 \mathrm{~d}$, and then methyl linoleate or methyl $\gamma$-linolenate was added to one culture as an albumin complex, which was prepared by sonicating $10 \mathrm{mg}$ of the fatty acid methyl ester with $1 \mathrm{ml}$ sterile $5 \%(\mathrm{w} / \mathrm{v})$ bovine serum albumin for $20 \mathrm{~min}$ at a concentration of $0.5 \mathrm{mg}$ ( $\mathrm{ml}$ culture broth $)^{-1}$. The values are means of two determinations. The control represents cells grown without the addition of fatty acids.

\begin{tabular}{|c|c|c|c|c|}
\hline \multirow[b]{3}{*}{ Fatty acid } & \multicolumn{4}{|c|}{ Fatty acid content $(\%, w / w)$} \\
\hline & \multicolumn{2}{|c|}{ Mut48 } & \multicolumn{2}{|c|}{ Mut49 } \\
\hline & Control & $+18: 2$ & Control & $+18: 3 \gamma$ \\
\hline $16: 0$ & $14 \cdot 6$ & $12 \cdot 2$ & $16 \cdot 3$ & $13 \cdot 8$ \\
\hline $18: 0$ & $7 \cdot 4$ & 6.9 & 11.8 & $11 \cdot 3$ \\
\hline $18: 1$ & 54.5 & $54 \cdot 2$ & $10 \cdot 7$ & $18 \cdot 4$ \\
\hline $18: 2$ & - & - & $43 \cdot 0$ & $32 \cdot 4$ \\
\hline $18: 2 \omega 9$ & $10 \cdot 9$ & $11.8+$ & - & - \\
\hline $18: 3 \gamma / 20: 0^{*}$ & 0.8 & 1.0 & $1 \cdot 7$ & $2 \cdot 7$ \\
\hline $20: 1$ & $2 \cdot 3$ & $2 \cdot 7$ & 0.6 & $1 \cdot 1$ \\
\hline $20: 2$ & - & - & 1.8 & $2 \cdot 4$ \\
\hline $20: 2 \omega 9$ & 1.6 & $2 \cdot 0$ & - & - \\
\hline $20: 3 \omega 9$ & $3 \cdot 7$ & 3.8 & - & - \\
\hline DGLA $/ 22: 0^{*}$ & 1.6 & $1 \cdot 1$ & 1.8 & 1.7 \\
\hline $20: 3$ & - & - & 5.0 & 3.4 \\
\hline Ara & - & $2 \cdot 2$ & $5 \cdot 0$ & $9 \cdot 5$ \\
\hline $24: 0$ & $2 \cdot 6$ & $2 \cdot 1$ & $2 \cdot 3$ & $3 \cdot 3$ \\
\hline
\end{tabular}

- , Undetectable.

* These pairs of fatty acids were not separated under the GLC conditions used. Both were present as minor components, as judged on reverse phase HPLC, in all cases except in Mut48 grown without linoleic acid addition, in which only 20:0 and 22:0 were found.

† Includes linoleic acid.

$\Delta 5$-desaturation (Shimizu et al., 1991b). Therefore, Mut44 was considered to be a mutant with low $\Delta 5$ desaturase activity. The fatty acid composition of Mut48 included 18:1 $(49.5 \%)$ as the most abundant fatty acid component and there was no fatty acid of the $\omega-6$ family in its mycelia. It was striking that several fatty acids of the $\omega-9$ family were accumulated. The $C_{20: 3}$ fatty acid was identified as 5,8,11-cis-eicosatrienoic acid $(20: 3 \omega 9)$ by comparing its mass spectra and proton NMR with those of the authentic compound. The remainder were identified on the basis of proton NMR and mass spectra of the corresponding picolinyl esters as 6,9-cis-octadecadienoic acid $(18: 2 \omega 9)$ and 8,11-cis-eicosadienoic acid $(20: 2 \omega 9)$. In Mut 49 , it was found that $46 \%$ of the total mycelial fatty acids was 18:2 and that the amounts of downstream fatty acid metabolites, i.e. 18:3 $\gamma$, DGLA and Ara, were very small compared with those in the case of the wild-type. As shown in Table 1, Mut48 produced Ara on cultivation in medium supplemented with $18: 2$.
Similarly, the percentage content of Ara in Mut49 was double that of the control on growth in a medium supplemented with $18: 3 \gamma$. These observations suggested that Mut48 is only defective in $\Delta 12$-desaturase, the other elongation and desaturation activities being retained, and that only $\Delta 6$-desaturase in Mut49 was defective.

\section{Lipid composition}

The lipid composition of the mutants and the wild type grown at 28 and $12^{\circ} \mathrm{C}$ are compared in Table 2 . The major lipids of all strains were triglyceride (TG), phosphatidylcholine (PC), phosphatidylethanolamine (PE) and phosphatidylserine (PS). In the wild type grown at $28{ }^{\circ} \mathrm{C}$, the relative content of TG was $82 \mathrm{~mol} \%$, and that of PC, PE and PS was 7,8 and $3 \mathrm{~mol} \%$, respectively. The lipid composition of Mut44 and Mut49 were almost the same as that of the wild type, but that of Mut48 included a somewhat higher ratio of phospholipids. At $12^{\circ} \mathrm{C}$, all strains contained a somewhat higher ratio of phospholipids, as compared with that in cells grown at $28^{\circ} \mathrm{C}$.

\section{Fatty acid composition on growth at low temperature}

The fatty acid composition of the mutants and wild type grown at 28 or $12^{\circ} \mathrm{C}$ is given in Table 3 . Regardless of the growth temperature, the wild type contained the highest level of Āra (44-46\%, w/w), whilst Mut44, Mut48 and Mut49 contained the highest levels of DGLA (32-34\%), $18: 1(46-50 \%)$ and $18: 2(25-32 \%)$, respectively. Mut44, like the wild type, accumulated several fatty acids of the $\omega$-3 family, i.e. 6,9,12,15-cis-octadecatetraenoic acid (18:4), 8,11,14,17-cis-eicosatetraenoic acid $(20: 4 \omega 3)$ and EPA, when grown at $12^{\circ} \mathrm{C}$. It is notable that the percentage content of $20: 4 \omega 3$ in Mut44 $(6.0 \%)$ was much higher than that in the case of the wild type $(0.4 \%)$, whilst EPA was decreased. In Mut48, there was no significant change in the fatty acid components when grown at a lower temperature, except for a marked increase in the $20: 3 \omega 9$ content from $9.3 \%$ at $28{ }^{\circ} \mathrm{C}$ to $18.2 \%$ at $12{ }^{\circ} \mathrm{C}$. In addition to the high content of $18: 2$, Mut49 showed some interesting changes in its fatty acid profile, as follows. Firstly, there was an unknown fatty acid, which was considered from mass spectra to be a $\mathrm{C}_{20: 3}$ fatty acid. However, its retention time on GLC was different from those of DGLA and 20:3 $\omega 9$. Secondly, the proportion of Ara increased from 6.3 to $22.7 \%$ at $12^{\circ} \mathrm{C}$. Thirdly, there was an additional $\mathrm{C}_{20: 4}$ fatty acid when it was grown at $12^{\circ} \mathrm{C}$. This fatty acid was entirely separated from Ara and 20:4 33 on high-resolution capillary GLC (Roscot Silar-5CP, $0.25 \mathrm{~mm} \times 50 \mathrm{~m}$; Nihon Chromato Works) (data not shown). The structures of these unknown PUFAs are being determined. 
Table 2. Lipid composition of the fatty acid desaturation-defective mutants of $M$. alpina $1 S-4$

All strains were grown in medium GY at either $28^{\circ} \mathrm{C}$ for $7 \mathrm{~d}$ or $12^{\circ} \mathrm{C}$ for $10 \mathrm{~d}$. The values are means of two independent determinations. Other minor components such as sterols, steroyl esters and glycolipids were not included for the calculation.

\begin{tabular}{|c|c|c|c|c|c|c|c|c|}
\hline \multirow[b]{3}{*}{ Lipids } & \multicolumn{8}{|c|}{ Lipid composition (mol \%) } \\
\hline & \multicolumn{4}{|c|}{$12^{\circ} \mathrm{C}$} & \multicolumn{4}{|c|}{$28^{\circ} \mathrm{C}$} \\
\hline & $1 \mathrm{~S}-4$ & Mut44 & Mut48 & Mut49 & $1 S-4$ & Mut44 & Mut48 & Mut49 \\
\hline TG & 76 & 80 & 66 & 72 & 82 & 84 & 76 & 83 \\
\hline PE & 10 & 8 & 14 & 10 & 7 & 6 & 9 & 6 \\
\hline PC & 10 & 9 & 14 & 13 & 8 & 7 & 11 & 8 \\
\hline PS & 4 & 3 & 6 & 5 & 3 & 3 & 4 & 3 \\
\hline
\end{tabular}

Table 3. Comparison of fatty acid composition of the fatty acid desaturationdefective mutants of $M$. alpina $1 S-4$

All strains were grown in medium GY at either $28^{\circ} \mathrm{C}$ for $7 \mathrm{~d}$ or at $12{ }^{\circ} \mathrm{C}$ for $6 \mathrm{~d}$ after preincubation for $1 \mathrm{~d}$ at $28^{\circ} \mathrm{C}$. All values are means of two determinations.

\begin{tabular}{|c|c|c|c|c|c|c|c|c|}
\hline \multirow[b]{3}{*}{ Fatty acid } & \multicolumn{8}{|c|}{ Fatty acid content $(\%, w / w)$} \\
\hline & \multicolumn{4}{|c|}{$12^{\circ} \mathrm{C}$} & \multicolumn{4}{|c|}{$28^{\circ} \mathrm{C}$} \\
\hline & $1 S-4$ & Mut44 & Mut48 & Mut49 & $1 S-4$ & Mut44 & Mut48 & Mut49 \\
\hline $16: 0$ & 8.6 & 6.9 & 4.6 & 5.8 & $11 \cdot 1$ & 10.8 & 9.9 & $12 \cdot 7$ \\
\hline $18: 0$ & 8.9 & 9.9 & 8.0 & $10 \cdot 2$ & $5 \cdot 3$ & 7.8 & $6 \cdot 1$ & 10.7 \\
\hline $18: 1$ & $10 \cdot 3$ & 11.8 & $46 \cdot 4$ & $8 \cdot 7$ & $13 \cdot 7$ & $11 \cdot 1$ & $49 \cdot 3$ & $15 \cdot 3$ \\
\hline $18: 2$ & $5 \cdot 4$ & $4 \cdot 2$ & - & $25 \cdot 5$ & $7 \cdot 4$ & 5.6 & - & $31 \cdot 8$ \\
\hline $18: 2 \omega 9$ & - & - & $12 \cdot 1$ & - & - & - & $12 \cdot 1$ & - \\
\hline $18: 3 \gamma / 20: 0^{*}$ & 4.9 & 9.5 & 0.7 & $2 \cdot 3$ & 4.6 & 9.6 & 0.7 & 1.7 \\
\hline $18: 3 \alpha / 20: 1 \dagger$ & 0.9 & $1 \cdot 4$ & 3.6 & $2 \cdot 7$ & $1 \cdot 1$ & $1 \cdot 1$ & $2 \cdot 7$ & $1 \cdot 0$ \\
\hline $18: 4$ & $0 \cdot 2$ & 1.0 & - & - & - & - & - & - \\
\hline $20: 2$ & 1.0 & $1 \cdot 1$ & - & $6 \cdot 1$ & 0.6 & 1.0 & - & $2 \cdot 1$ \\
\hline $20: 2 \omega 9$ & - & - & $3 \cdot 2$ & - & - & - & $2 \cdot 1$ & - \\
\hline DGLA/22:0* & $7 \cdot 8$ & 33.9 & 1.5 & 1.7 & $6 \cdot 5$ & $32 \cdot 8$ & $2 \cdot 2$ & $2 \cdot 9$ \\
\hline $20: 3 \omega 9$ & - & - & $18 \cdot 2$ & - & - & - & $9 \cdot 3$ & - \\
\hline $20: 3 \ddagger$ & - & - & - & $6 \cdot 4$ & - & - & - & $7 \cdot 8$ \\
\hline $20: 3 \omega 3 /$ Ara $\$$ & $45 \cdot 4$ & $8 \cdot 1$ & - & $22 \cdot 7$ & $44 \cdot 4$ & $11 \cdot 7$ & - & $6 \cdot 3$ \\
\hline $20: 4 \omega 3$ & 0.4 & 6.0 & - & - & - & - & - & - \\
\hline $20: 4 \ddagger$ & - & - & - & $3 \cdot 2$ & - & - & - & - \\
\hline $24: 0$ & $2 \cdot 2$ & 3.9 & $1 \cdot 7$ & $3 \cdot 3$ & $5 \cdot 2$ & $8 \cdot 5$ & $5 \cdot 6$ & $7 \cdot 8$ \\
\hline EPA & $4 \cdot 1$ & $2 \cdot 4$ & - & 1.5 & - & - & - & - \\
\hline
\end{tabular}

-, Undetectable.

* Both were found in all strains except for Mut48, in which 18:3r and DGLA were not detected.

† Only $20: 1$ was found in all strains on growth at $28^{\circ} \mathrm{C}$ but $18: 3 \alpha$ was found as an additional fatty acid on growth at $12{ }^{\circ} \mathrm{C}$, except in the case of Mut48, in which $18: 3 \alpha$ was not detected even at this temperature. $\ddagger$ These fatty acids were determined to be eicosatrienoic acid $(20: 3)$ and eicosatetraenoic acid $(20: 4)$, respectively, but the positions of the double bonds have not yet been determined.

$\S$ Trace amounts of $20: 3 \omega 3$ were detected on growth at $12^{\circ} \mathrm{C}$.

\section{Growth characteristics and fatty acid content}

Each of the three mutants was inoculated as a spore suspension into medium GY and grown at either 28 or $12^{\circ} \mathrm{C}$ after preincubation at $28^{\circ} \mathrm{C}$ for $1 \mathrm{~d}$. All the mutants grew somewhat more slowly, especially at $12^{\circ} \mathrm{C}$, than the wild type (Fig. 2). At $12^{\circ} \mathrm{C}$ the lag phase for the wild type was about $1 \mathrm{~d}$, whilst that for the mutants were about $2 \mathrm{~d}$. The total mycelial fatty acid content corresponded to the growth rate, but reached a plateau more 


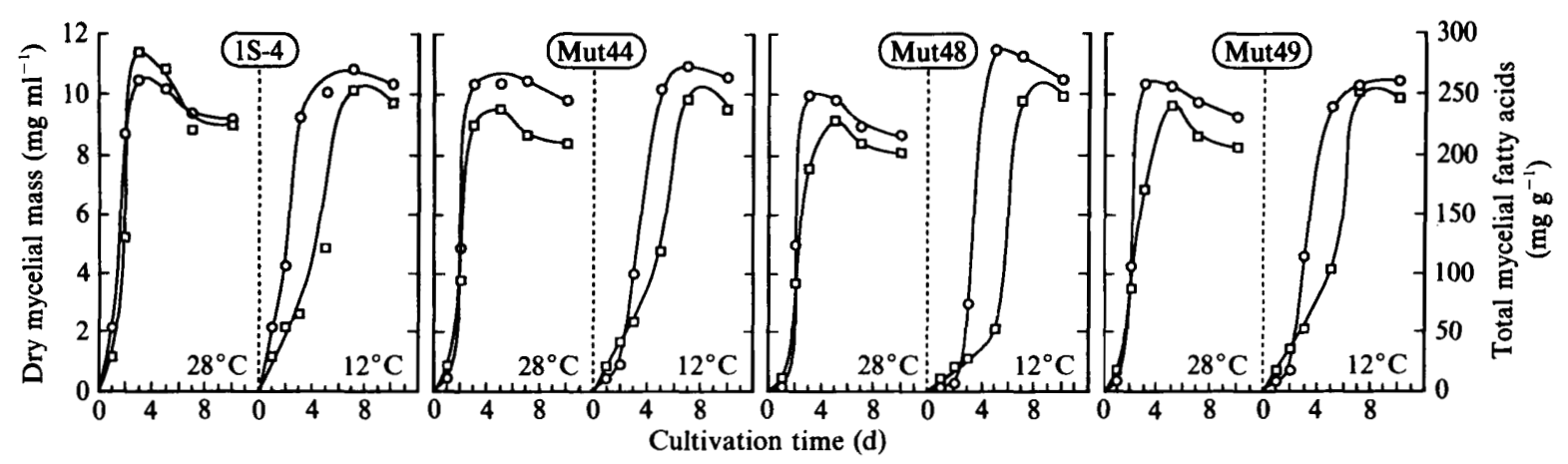

Fig. 2. Growth and total mycelial fatty acid content of $M$. alpina $1 \mathrm{~S}-4$ and mutants. Growth $(O)$ is expressed as mg dry mycelial mass (ml culture broth $)^{-1}$ and total mycelial fatty acids $(\square)$ are expressed as $\mathrm{mg}$ fatty acids ( $\mathrm{g}$ dry mycelial mass) $)^{-1}$. All values shown are means of two determinations.

slowly than the growth rate. All mutants accumulated almost the same level of fatty acids when grown at $28^{\circ} \mathrm{C}$ for $5 \mathrm{~d}$ or at $12^{\circ} \mathrm{C}$ for $7 \mathrm{~d}$.

\section{Discussion}

As far as we know, this paper is the first to report the derivation of mutants defective in fatty acid desaturation of an Ara-producing fungus. Based on the fatty acid profiles, the mutants reported here are considered to be defective in $\Delta 5$-, $\Delta 12$ - and $\Delta 6$-desaturation. In Mut44 and Mut49, which are considered to be defective in $\Delta 5$ - and $\Delta 6$-desaturation, respectively, Ara still existed. The occurrence of Ara may be explained by either of the following assumptions. The explanation for the lack of desaturation in the mutants is that mutation could occur in the genes encoding desaturases. It is possible that the $\Delta 5$ - or $\Delta 6$-desaturase produced from the respective modified gene still retained some activity. Another possible assumption is that there could be two kinds of desaturases for desaturation at one position in the fatty acids, i.e. one requiring acyl-CoA ester and another requiring lipid-linked fatty acids (Pugh \& Kates, 1977; Kamisaka et al., 1990). The formation of $20: 3 \omega 9$ in Mut48 would occur through the same sequential reactions suggested by Fulco \& Mead (1959), i.e. 18:1 was desaturated at the $\Delta 6$-position into $18: 2 \omega 9$ followed by elongation and $\Delta 5$-desaturation to $20: 3 \omega 9$. The reason for the marked increases in the levels of $20: 3 \omega 9$ and Ara in Mut48 and Mut49, respectively, on the temperature shift to $12{ }^{\circ} \mathrm{C}$ (Table 3), is still unclear, but it is possible that $\Delta 6$-desaturase becomes more active at low temperature, or that the mutation of $\Delta 6$-desaturation in Mut49 was temperature sensitive.

It has been suggested that one of the roles of PUFAs is the maintenance of the proper fluidity of biological membranes, especially at low temperatures (Chapman, 1975). Though the mutants reported here germinated somewhat slower than the wild type at low temperature $\left(12^{\circ} \mathrm{C}\right.$; data not shown), they grew as well as the wild type. This observation may suggest that Ara and other $\omega-6$ fatty acids are not absolutely required for normal growth of this fungus, and that the other unusual PUFAs, such as $20: 3 \omega 9$ in Mut48 or the unknown fatty acids in Mut49, may compensate for their requirement of fatty acids for the maintenance of proper membrane fluidity.

Like the wild type, all the mutants accumulated a relatively high level of total fatty acids, and therefore they are promising with regard to their use in the fermentative production of fatty acids. Also, these mutants may be useful for cloning of genes encoding fungal-type desaturases by means of complementation of the mutants.

We wish to thank Mr Hiroshi Kawashima of Suntory Ltd for performing the mass spectrometry and proton NMR. This work was supported in part by a Grant-in-Aid from the Ministry of Education, Science and Culture of Japan.

\section{References}

Chapman, D. (1975). Phase transitions and fluidity characteristics of lipids and cell membranes. Quarterly Reviews of Biophysics 8, 185235.

Christie, W. W., Brechany, E. Y., Johnson, S. B. \& Holman, R. T. (1986). A comparison of pyrolidide and picolinyl ester derivatives for the identification of fatty acids in natural samples by gas chromatography-mass spectrometry. Lipids 21, 657-661.

FulCo, A. J. \& Mead, J. F. (1959). Metabolism of essential fatty acids; VIII. Origin of 5,8,11-cis-eicosatrienoic acid in the fat-deficient rat. Journal of Biological Chemistry 234, 1411-1416.

Kamisara, Y., Yokoch, T., Nakahara, T. \& Suzuki, O. (1990). Incorporation of linoleic acid and its conversion to $\gamma$-linolenic acid in fungi. Lipids 25, 54-60. 
Pugh, E. L. \& Kates, M. (1977). Direct desaturation of eicosatrienoyl lecithin to arachidonyl lecithin by rat liver microsomes. Journal of Biological Chemistry 252, 68-73.

Shimizu, S., Kawashima, H., Shinmen, Y., Akimoto, K. \& Yamada, H. (1988a). Production of eicosapentaenoic acid by Mortierella fungi. Journal of American Oil Chemists' Society 65, 1455-1459.

Shmizu, S., Shinmen, Y., Kawashma, H., Akimoto, K. \& Yamada, H. (1988b). Fungal mycelia as a novel source of eicosapentaenoic acid, activation of enzyme(s) involved in eicosapentaenoic acid production at low temperature. Biochemical and Biophysical Research Communications 150, 335-341.

Shimizu, S., Akimoto, K., KaWashima, H., Shimmen, Y. \& Yamada, H. (1989). Production of dihomo- $\gamma$-linolenoic acid by Mortierella alpina 1S-4. Journal of American Oil Chemists' Society 66, 237241.

Shimizu, S., Jareonkitmongkol, S., Kawashima, H., Akimoto, K. \& YAMADA, H. (1991a). Production of a novel $\omega 1$-eicosapentaenoic acid by Mortierella alpina 1S-4 grown on 1-hexadecene. Archives of Microbiology 156, 163-166.

Shimizu, S., Akimoto, K., Shinmen, Y., KaWashima, H., Sugano, M. \& YAMADA, H. (1991 b). Sesamin is a potent and specific inhibitor of $\Delta 5$-desaturase in polyunsaturated fatty acid biosynthesis. Lipids 26 , $512-516$.

Shinmen, Y., Shmizu, S., Akimoto, K., KaWashima, H. \& Yamada, H. (1989). Production of arachidonic acid by Mortierella fungi: selection of a potent producer and optimization of culture conditions for large scale production. Applied Microbiology and Biotechnology 31, 11-16.

Wada, H. \& Murata, N. (1989). Synechocystis PCC6803 mutants defective in desaturation of fatty acids. Plant and Cell Physiology 30 , 971-978.

Yamada, H., Shimizu, S. \& Shinmen, Y. (1987). Production of arachidonic acid by Mortierella elongata 1S-5. Agricultural and Biological Chemistry 51, 785-790. 